blick auf $\int 52$ Abs. 24a EStG a. F. herausgestellte besondere Vertrauenswirkung erstreckte sich nur auf den Zeitraum bis zur Zuleitung des Regierungsentwurfs für das StEntlG 1999/2000/2002 an den Bundesrat (am 20.11.1998) und wirkt - wie dem Beschluss eindeutig zu entnehmen ist nur im Zusammenhang mit einer bindenden Dispositionsentscheidung des Steuerpflichtigen, die bis zu diesem Zeitpunkt verwirklicht worden ist. Daran fehlt es im Streitfall.

c) Der Senat teilt auch nicht die Auffassung des K1., dass der Gesetzgeber nach Einführung der Altersgrenze für Kassenärzte durch $\int 95$ Abs. 7 SGB V i.d.F. des Gesundheitsstrukturgesetzes mit Wirkung zum 1.1.1999 daran gehindert gewesen sei, speziell für niedergelassene Ärzte mit Zulassung als Vertragsarzt den halben Steuersatz für Gewinne aus der Veräußerung der Kassenarztpraxis übergangslos abzuschaffen. Entsprechendes ergibt sich entgegen der Auffassung des Kl. nicht aus der Begründung des Gesundheitsstrukturgesetzes. Der Gesetzgeber ist davon ausgegangen, dass Ärzte und Zahnärzte in der Regel spätestens mit Vollendung des 65. Lebensjahres über eine ausreichende Altersversorgung, vor allem durch die berufsständischen Versorgungswerke verfügen (vgl. BT-Dr. 12/3209, S. 49). Daraus ergibt sich jedoch nicht, dass der Gesetzgeber die Besteuerung des Gewinns aus der Veräußerung der Arztpraxis mit dem halben Steuersatz als für die Altersversorgung der Ärzte und Zahnärzte bedeutsam angesehen hat. Das BVerfG hat entschieden, dass die Einführung der Altersgrenze mit der Verfassung vereinbar war (BVerfG-Beschluss in NJW 1998, 1776). In der Entscheidung hat das BVerfG maßgeblich darauf abgestellt, dass den bereits zugelassenen Ärzten die Möglichkeit eingeräumt wurde, wenigstens 20 Jahre lang eine vertragsärztliche Praxis zu betreiben. Der Gesetzgeber habe davon ausgehen dürfen, dass diese Zeitspan- ne ausreiche, um getätigte Investitionen zu erwirtschaften und eine angemessene Alterssicherung aufzubauen. Auf die Fortgeltung des halben Steuersatzes für Gewinne aus der Veräußerung der Arztpraxis hat auch das BVerfG nicht abgestellt.

Im Streitfall ist auch weder vorgetragen noch ersichtlich, dass die ehemalige Kl. eine bestimmte Besteuerung des Veräußerungsgewinns in ihre Altersvorsorgeplanung einbezogen hatte. Soweit die Besteuerung mit dem halben Steuersatz aber nachweislich konkret in das Konzept der Altersversorgung einbezogen worden ist und der Wegfall dieses Steuervorteils zu einer gravierenden Gefährdung der Altersversorgung geführt haben sollte, kommt nach dem BFH-Beschluss in BFH/NV 2003, 777, ein Erlass der Steuer aus Billigkeitsgründen in Betracht. Dem schließt sich der erkennende Senat an. Die Frage des Erlasses kann im Rahmen der Klage gegen den Einkommensteuerbescheid allerdings nicht geprüft werden.

d) Unerheblich ist, dass die ehemalige K1. der Besteuerung des Veräußerungsgewinns zum normalen Tarif mit Rücksicht auf die Dauer des Nachbesetzungsverfahrens nicht ausweichen konnte. Der Senat geht insofern mit dem K1. in tatsächlicher Hinsicht davon aus, dass eine bindende Entscheidung über die Veräußerung der Arztpraxis bis zum Inkrafttreten des StEntlG 1999/2000/2002 auch dann nicht hätte herbeigeführt werden können, wenn das Nachbesetzungsverfahren unmittelbar nach dem Bekanntwerden der Steueränderungspläne am 20.11.1998 eingeleitet worden wäre. Darauf kommt es jedoch nicht an. Die verfassungsrechtliche Zulässigkeit einer Rechtsänderung bestimmt sich nicht danach, ob der Steuerpflichtige ihr noch rechtzeitig ausweichen kann, sondern ob sie sachlich gerechtfertigt ist.

BUCHBESPRECHUNGEN

DOI: 10.1007/s00350-010-2716-z

\section{Medizinprodukte-Recht. Kommentar.}

Von Gert Schorn, Hans Georg Baumann, Annika S. Bien, Volker Lücker und Heike Wachenhausen. Deutscher Apotheker Verlag, Stuttgart, Loseblatt in 4 Ordnern, Gesamtwerk 4744 S., hier: 25. Erg.-Lfg. 2010

Der gesetzgeberische Fleiß im Medizinprodukte- und Arzneimittelrecht macht zeitnahe Aktualisierungen in kurzen Intervallen erforderlich. Zum Nutzen des Lesers haben Herausgeber und Autoren des Kommentars die Änderungen durch das 4. MPGÄndG rasch in das Werk übernommen. Der Kommentar enthält die amtlichen Begründungen zu den Änderungen des Medizinproduktegesetzes, der Medizinprodukte-Verordnung, der Medizinprodukte-Sicherheitsplanverordnung, der Medizinprodukte-Betreiberverordnung und der Medizinprodukte-Gebührenverordnung. Im Kommentar wird hinsichtlich des neuen Rechts auf entsprechende Veröffentlichungen im Medizinprodukte-Journal (MPJ) der Jahre 2007-2009 verwiesen. Über diesen Link stehen dem Leser des Kommentars weitere Informationen und Hintergrundwissen zu diesen Themen offen.

Die geänderten medizinprodukterechtlichen Vorschriften werden nach alter und neuer Rechtslage gegenübergestellt. Wie bereits in der Vorlieferung (Textvergleich der geänderten europäischen Medi-

Rechtsanwalt und Arzt Dr. med. Christian Jäkel,

Fachanwalt für Medizinrecht,

Lübben, Deutschland zinprodukterichtlinien) werden hier die ab dem 21.3.2010 geltenden Vorschriften in Textkästen hervorgehoben. Die ab diesem Zeitpunkt entfallenen alten Vorschriften sind wiederum unterstrichen dargestellt. Damit ist für den Leser leicht erkennbar, welche Vorschriften ab dem 21.3.2010 gelten. Diese bereits bewährte Darstellungsweise erleichtert den Überblick erheblich.

Im Bereich aktualisierter Gesetzestexte ist der Abdruck der 15. AMG-Novelle hervorzuheben. Darüber hinaus werden Aktualisierungen der Arzneimittelfarbstoffverordnung, des Infektionsschutzgesetzes, des Transfusionsgesetzes und des Heilmittelwerbegesetzes in konsolidierter Fassung abgedruckt. Außerdem wurde der Text des Akkreditierungsstellengesetzes aufgenommen, das im Sommer 2009 aufgrund des entsprechenden Maßnahmenpaketes der EU für den freien Warenverkehr erlassen werden musste. Statt über 20 Akkreditierungsstellen wird es künftig in Deutschland lediglich die Deutsche Akkreditierungsstelle (DAkkS) geben.

Auf europäischer Ebene rundet die 25. Aktualisierungslieferung den Kommentar ab mit dem interpretatorischen Dokument der EUKommission zur Implementierung der Richtlinie 2007/47/EG, der Verordnung (EG) Nr. 764/2008, verschiedenen MEDDEV-Dokumenten und dem Manual on borderline and classification für Medizinprodukte, erstellt von der bei der EU-Kommission angesiedelten Medical Devices Expert Group on borderline and classification.

Aktualisiert wurden die Inhaltsverzeichnisse und das Sachverzeichnis. Letzteres enthält alle wesentlichen Suchstichworte und stellt eine wertvolle Arbeitshilfe dar.

Besonders nutzbringend ist die beiliegende CD-ROM mit Auszügen aus dem Kommentar, auf der der Leser eine sehr gelungene Übersicht zum Recht der Medizinprodukte, eine Vielzahl aktueller Gesetzestexte, das Medizinprodukterecht der Schweiz und Österreichs, sämtliche Gerichtsentscheidungen sowie das Sachverzeichnis findet. Die Inhalte lassen sich gut mit Suchfunktionen recherchieren. 